\title{
Paper The Optimum-efficiency Beam Multiplier for an Arbitrary Number of Output Beams and Power Distribution
}

\author{
Fabrizio Frezza ${ }^{1}$, Marian Marciniak $^{2}$, and Lara Pajewski ${ }^{1}$ \\ ${ }^{1}$ Department of Information Engineering, Electronics and Telecommunications, Sapienza University of Rome, Rome, Italy \\ ${ }^{2}$ National Institute of Telecommunications, Warsaw, Poland
}

https://doi.org/10.26636/jtit.2017.118617

\begin{abstract}
This paper deals with phase gratings working in the paraxial domain. The profile of the optimum-efficiency beam multiplier with an arbitrary number of output diffraction orders is derived in an analytic form by exploiting methods from the calculus of variation. The output beams may be equi-intense or with arbitrary distribution of power. Numerical examples are given for different values of the number of output beams.
\end{abstract}

Keywords—beam multipliers, diffractive optical elements, gratings, periodic structures.

\section{Introduction}

Diffractive Optical Elements (DOEs) [1] that split a laser beam into multiple output beams are used in many industrial and scientific applications, i.e. in optical signal processing, laser manufacturing, interferometry, read-write magneto-optic data-storage systems, and optical networks. In several cases, the so-called polarizing beam splitters are used [2], which divide an incoming beam into output beams with different polarization states. More often, beam multipliers are employed to replicate an incoming beam into a set of output beams with identical polarization state. The output beams may be equi-intense or show a suitable distribution of power. To realize a beam multiplier, binary Dammann structures are primarily used [3], [4]. Continuous-phase gratings are also sometimes realized [5]-[7].

The diffraction efficiency of a beam multiplier is the fraction of the incident beam power that is converted into the power of the desired output beams. Maximization of this parameter is a fundamental target in designing beam multipliers. Usually, in the evaluation of the diffraction efficiency, absorption and reflection losses are not considered. The former is negligible and the latter can be reduced by using suitable antireflection coatings.

Another important goal to be pursued in the design of beam multipliers is the uniformity of the output beams (or the achievement of the desired power distribution).

The upper bound of the diffraction efficiency of beam multipliers was derived and studied in [10]-[12], for diffractive phase gratings working in the scalar domain. Obviously, the maximum diffraction efficiency of real devices is generally lower than the upper bound. It is worth citing that, by exploiting the further degree of freedom offered by the polarization, in some cases it is possible to overcome the efficiency upper bound and achieve 100\% efficiency [13]. In this paper, we focus on phase gratings working in the paraxial domain. We exploit mathematical methods from the calculus of variations for solving the problem of finding optimal profiles for diffractive beam multipliers and we calculate their diffraction efficiency. The problem of optimizing a phase profile is usually faced by using numerical techniques [8], [9], whereas here the optimal profiles in an analytic form are derived.

The present work is the continuation of previous studies, where our research team concentrated on beam multipliers with three [5], [14] and four [6] equi-intense output beams. In particular, in [5], the profile of a DOE producing three equi-intense diffraction orders with the maximum efficiency was derived in an analytic form. In [14], a full-wave electromagnetic analysis of this beam multiplier was performed. In [6], the profile of a DOE producing four equi-intense diffraction orders with the maximum efficiency was derived and its full-wave electromagnetic analysis was presented. It is worth mentioning that in [15] the optimum triplicator proposed in [5] was realized, experimentally tested, and compared to binary gratings of Dammann type. Here, we generalize the procedure proposed in [5], [6] and prove that an optimum-efficiency beam multiplier with an arbitrary number of equi-intense diffraction orders exists. We derive its phase transmittance in an analytic form. This phase transmittance can also be used to design the optimum-efficiency beam multiplier with a fixed power distribution.

The paper is organized as follows. In Section 2 the analytical derivation of the phase transmittance of the optimumefficiency beam multiplier is presented. In Section 3 numerical examples for different values of the number of output beams are presented. For each considered number of output beams, we give the profile of the optimum beammultiplier and calculate its diffraction efficiency. Conclusions and ideas for future work are given in Section 4. 


\section{Theory}

As is well known, in the paraxial domain the vectorial nature of light can be neglected and the influence of a diffractive element on the illuminating wavefront can be described by its transmission function [16]. Here the transmission function of a phase grating producing an arbitrary number of replicas of an incident field is obtained, with an arbitrary power distribution and with the maximum diffraction efficiency (see Fig. 1).

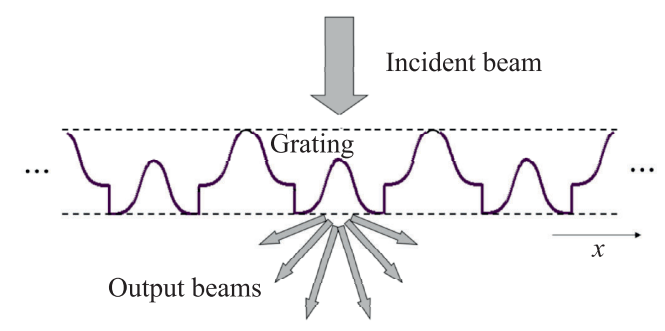

Fig. 1. Scheme illustrating the operation of a phase grating acting as a beam multiplier.

Let us consider a grating having the transmission function $\tau(x)=\mathrm{e}^{i \Phi(x)}$, where $\Phi(x)$ denotes the phase profile. Without loss of generality, in the following formulas we assume a unitary value (in suitable units) of the grating period $d$. Due to the periodicity of the structure, the following Fourier series expansion holds:

$$
\tau(x)=\sum_{\mathrm{m}=-\infty}^{\infty} \tau_{\mathrm{m}} \mathrm{e}^{i 2 \pi \mathrm{m} x},
$$

where $\tau_{\mathrm{m}}$ is the Fourier coefficient corresponding to the diffracted order of index $m$ having the following expression:

$$
\tau_{\mathrm{m}}=\int_{-\frac{1}{2}}^{\frac{1}{2}} \mathrm{e}^{i[\Phi(x)-2 \pi \mathrm{m} x]} \mathrm{d} x
$$

We define $M$ as the set of indices corresponding to the desired diffracted orders. Generalizing the approach proposed in [5], [6], we consider the functional:

$$
\mathscr{I}=\sum_{\mathrm{m} \in \mathrm{M}} \beta_{\mathrm{m}}\left|\tau_{\mathrm{m}}\right|^{2}
$$

where $\beta_{\mathrm{m}}$ are suitable positive multipliers and the sum includes the desired output diffracted orders. The first variation of $\mathscr{I}$ is

$$
\delta \mathscr{I}=\sum_{\mathrm{m} \in \mathrm{M}} \beta_{\mathrm{m}} \delta\left|\tau_{\mathrm{m}}\right|^{2}
$$

and the first variation of the Fourier-coefficient square magnitude is:

$$
\begin{gathered}
\delta\left|\tau_{\mathrm{m}}\right|^{2}= \\
=i \int \varepsilon(x)\left\{\tau_{\mathrm{m}}^{*} \mathrm{e}^{i[\Phi(x)-2 \pi \mathrm{m} x]}-\tau_{\mathrm{m}} \mathrm{e}^{-i[\Phi(x)-2 \pi \mathrm{m} x]}\right\} \mathrm{d} x,
\end{gathered}
$$

where the difference between a typical phase profile and the optimum one has been denoted by $\varepsilon(x)$. On imposing that the first variation of $\mathscr{I}$ vanishes, we have:

$$
\sum_{\mathrm{m} \in \mathrm{M}} \beta_{\mathrm{m}}\left|\tau_{\mathrm{m}}\right| \sin \left(\alpha_{\mathrm{m}}-\Phi(x)+2 \pi \mathrm{m} x\right)=0
$$

being $\alpha_{\mathrm{m}}$ the argument of $\tau_{m}$. From Eq. (6) it follows that

$$
\begin{aligned}
& \cos \Phi \sum_{\mathrm{m} \in \mathrm{M}} \beta_{\mathrm{m}}\left|\tau_{\mathrm{m}}\right| \sin \left(\alpha_{\mathrm{m}}+2 \pi \mathrm{m} x\right)= \\
& =\sin \Phi \sum_{\mathrm{m} \in \mathrm{M}} \beta_{\mathrm{m}}\left|\tau_{\mathrm{m}}\right| \cos \left(\alpha_{\mathrm{m}}+2 \pi \mathrm{m} x\right)
\end{aligned}
$$

Assuming that

$$
R(x)=\sum_{\mathrm{m} \in \mathrm{M}} \beta_{\mathrm{m}}\left|\tau_{\mathrm{m}}\right| \cos \left(\alpha_{\mathrm{m}}+2 \pi \mathrm{m} x\right)
$$

and

$$
\Gamma(x)=\sum_{\mathrm{m} \in \mathrm{M}} \beta_{\mathrm{m}}\left|\tau_{\mathrm{m}}\right| \sin \left(\alpha_{\mathrm{m}}+2 \pi \mathrm{m} x\right),
$$

Eq. (6) can be satisfied if $\Phi(x)$ satisfies

$$
\cos [\Phi(x)]=g(x) R(x)
$$

and

$$
\sin [\Phi(x)]=g(x) \Gamma(x),
$$

being $g(x)$ a real arbitrary function. Therefore, the phase distribution $\Phi_{\text {opt }}(x)$ maximizing the functional $\mathscr{I}$ assumes the form:

$$
\Phi_{\text {opt }}(x)=\arctan \left[\frac{\Gamma(x)}{R(x)}\right]+\pi \operatorname{step}[\mathrm{R}(\mathrm{x})],
$$

where $\operatorname{step}(\cdot)$ denotes the Heaviside step function, i.e., for $x \geq 0$ : $\operatorname{step}(x)=1$, for $x<0$ : $\operatorname{step}(x)=0$. The parameters $\alpha_{\mathrm{m}}$ and $\beta_{\mathrm{m}}$ have to be chosen in such a way that all the Fourier coefficients $\left|\tau_{m}\right|$ have the same magnitude, or else they respect a fixed distribution. In this case, a maximum of $\mathscr{I}$ is also a maximum of transmission efficiency and Eq. (12) gives the profile of the optimum-efficiency beam multiplier.

\section{Numerical Results}

Although the expression in Eq. (12) seems to be quite complicated, the optimum profile assumes, eventually apart from discontinuities, a simple and regular shape.

In Figs. 2 and 3, two different solutions for the four-beam multiplier are reported. The phase distribution $\Phi(x)$ is plotted as a function of $\frac{x}{d}$.

The first solution works on the diffracted orders $m= \pm 1$ and \pm 3 . In this case, for symmetry reasons $\alpha_{1}=\alpha_{-1}, \beta_{1}=\beta_{-1}$, $\alpha_{3}=\alpha_{-3}, \beta_{3}=\beta_{-3}$, it is possible to choose $\alpha_{1}=0$ and $\beta_{1}=1$. The following optimum values for the other two parameters are found: $\beta_{3}=1.046$ and $\alpha_{3}=1.845$. The achieved diffraction efficiency is $\eta=91.9025 \%$. This result is consistent with [6]. 


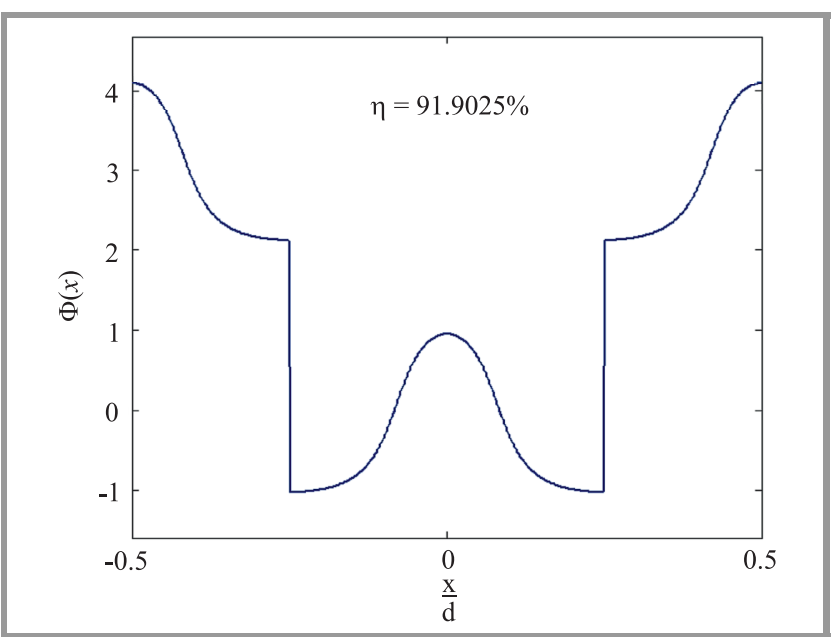

Fig. 2. Profile of the optimum 4-beam multiplier, working on the diffracted orders $m= \pm 1$ and \pm 3 .

The second solution works on the diffracted orders $m= \pm 1$ and \pm 2 . For symmetry reasons $\alpha_{1}=\alpha_{-1}, \beta_{1}=\beta_{-1}, \alpha_{2}=$ $\alpha_{-2}, \beta_{2}=\beta_{-2}$, it is possible to choose $\alpha_{1}=0$ and $\beta_{1}=1$. The following optimum values for the other two parameters are found: $\beta_{2}=1.05$ and $\alpha_{2}=1.5883$. The diffraction efficiency turns out to be $\eta=94.1306 \%$.

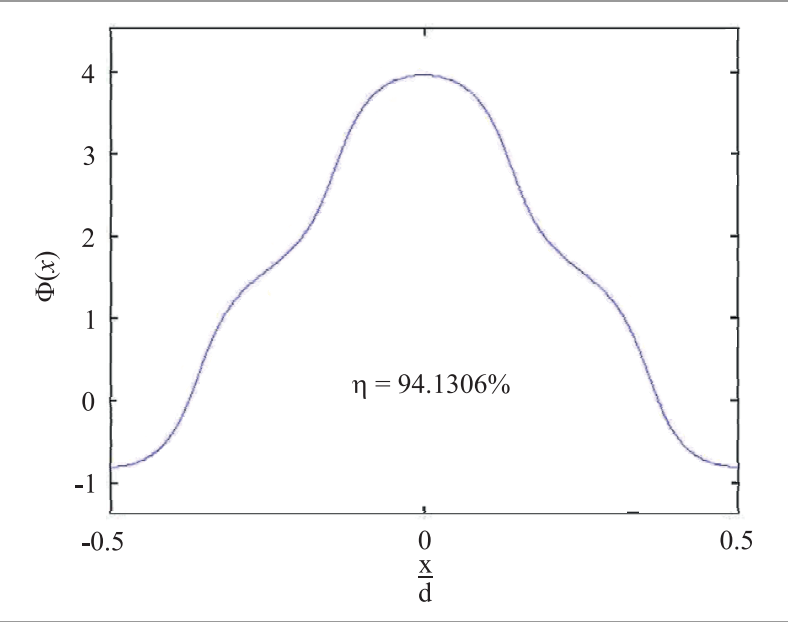

Fig. 3. Profile of the optimum 4-beam multiplier, working on the diffracted orders $m= \pm 1$ and \pm 2 .

These examples show that, for a given number of output beams, different solutions are possible if there are no specifications on the diffracted orders to be used. The second solution presents an efficiency higher than the first. However, in the first solution the output beams are equally spaced. Both the presented solutions are even-phase gratings. It is also possible to work on different diffracted orders obtaining a four-beam multiplier with asymmetric phase distribution. If the angular directions of the output beams are not specified, then as part of the optimization problem it is necessary to determine which diffracted orders have to be chosen to achieve the maximum transmission efficiency.
It is observed that the first solution presents a discontinuity in $x= \pm 0.25 d$, whereas the second solution is continuous. Note that, if a beam is splitted into an even number of odd diffracted orders, the optimal phase distribution will always have discontinuities.

In Fig. 4, a further example is presented. The profile of the optimum-efficiency five-beam multiplier is plotted as a function of $\frac{x}{d}$. This grating works on the diffracted orders $m=0, \pm 1$ and \pm 2 . For symmetry reasons $\alpha_{1}=\alpha_{-1}, \beta_{1}=$ $\beta_{-1}, \alpha_{2}=\alpha_{-2}, \beta_{2}=\beta_{-2}$, it is possible to choose $\alpha_{0}=0$ and $\beta_{0}=1$. The following optimum values for the other parameters are found: $\beta_{1}=0.459, \alpha_{1}=-1.5708, \beta_{2}=$ $0.899, \alpha_{2}=3.1416$. The diffraction efficiency turns out to be $\eta=92.1 \%$.

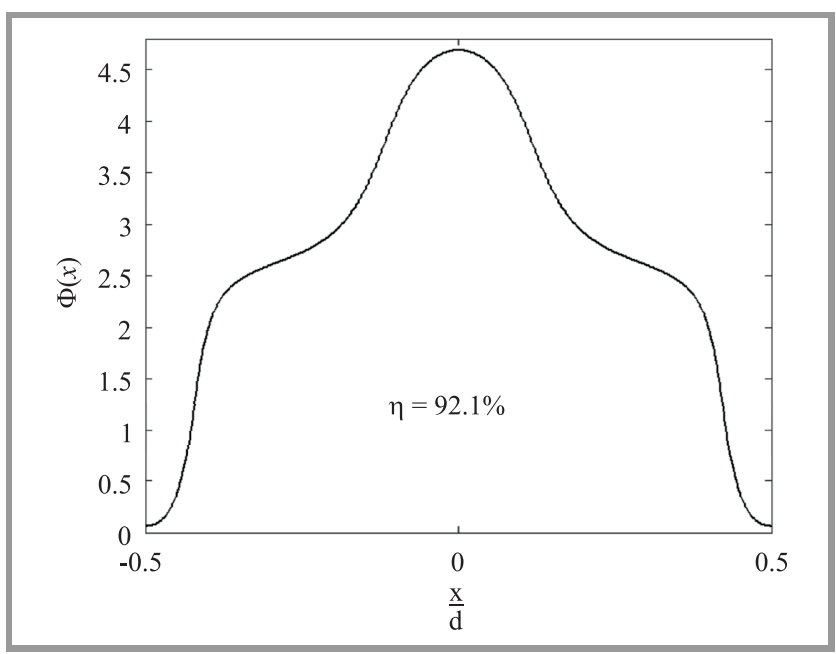

Fig. 4. Profile of the optimum 5-beam multiplier.

For each beam multiplier, the parameters $\alpha_{\mathrm{m}}$ and $\beta_{\mathrm{m}}$ maximizing the transmission efficiency have been found by imposing a beam uniformity equal to 0.001 and by performing a simple optimization search over a multi-dimensional grid of possible values for the parameters. For sure, there are better and faster ways of solving this optimization problem, but our aim was giving some numerical examples to highlight theoretical work. For high values of the number of output beams, this kind of optimization search becomes computationally difficult and more sophisticated techniques have to be applied to find the parameters $\alpha_{\mathrm{m}}$ and $\beta_{\mathrm{m}}$.

As already mentioned, we derived the profile of the optimum-efficiency beam multiplier in the paraxial domain. In the resonance domain, when grating-profile features have transverse dimensions comparable with the wavelength of the impinging radiation, the scalar diffraction theory fails. To study the properties of a DOE rigorously, a full-wave application of the electromagnetic theory is necessary. A rigorous treatment of the problem allows, for example, to understand the operational limits of a diffractive beam multiplier and study its angular response, as in [6] and [14]. The theory of propagation of electromagnetic waves in periodic media is well developed [17]-[21]. An efficient and versatile electromagnetic spectral-domain technique 
that we suggest to apply for the full-wave investigation of DOEs is the Fourier Modal Method. This approach was originally developed for the characterization of twodimensional diffraction gratings, but nowadays is employed also for the modeling of two-dimensional photonic crystals [22]-[25], crossed gratings and three-dimensional photonic crystals [26], as well as electromagnetic band-gap materials working in the microwave region of the frequency spectrum [27]-[33].

\section{Conclusions}

This paper focuses on the problem of splitting a beam into a set of equi-intense output beams, or in beams respecting a fixed power distribution. The profile of the optimumefficiency beam multiplier, with an arbitrary number of output diffraction orders, is derived in an analytic form; methods from the calculus of variation are exploited. $\mathrm{Nu}$ merical examples are given, for different values of the number of equi-intense output beams. For a given number of output beams, solutions with different angular directions of the output beams are compared.

Ideas for future research activities include:

- checking the performances of the optimum beam multiplier when a high number of output beams is required,

- performing a detailed comparison between the optimum beam multiplier and Dammann gratings,

- designing suitable antireflection coatings for the optimum beam multiplier and studying their effect on the performance of the grating,

- carrying out a full-wave study of the optimum beam multiplier by using a rigorous electromagnetic technique, such as the Fourier Modal Method,

- fabricating and measuring some prototypes.

\section{Acknowledgements}

The authors would like to thank Franco Gori for his useful suggestions and ideas, which were essential for the development of this work. This work benefited from the networking activities carried out in COST (European Cooperation in Science and Technology) Actions MP0702 "Towards Functional Sub-Wavelength Photonic Structures" (http://cost-mp0702.nit.eu) and TU1208 "Civil engineering applications of Ground Penetrating Radar" (www.GPRadar.eu). The paper was finalized during the TU1208 STSM no. 37998, carried out by Lara Pajewski at the National Institute of Telecommunications of Poland from 5 to 24 June 2017. The authors are grateful to COST for funding and supporting the Actions MP0702 and TU1208.

\section{References}

[1] G. J. Swanson, "Binary optics technology: the theory and design of multi-level diffractive optical elements", Tech. Rep. no. TR-854, Massachusetts Institute of Technology, Lexington Lincoln Lab., pp. 1-47, 1989 [Online]. Available: www.dtic.mil/dtic/tr/fulltext/ u2/a213404.pdf

[2] L. Pajewski, R. Borghi, G. Schettini, F. Frezza, and M. Santarsiero, "Design of a binary grating with subwavelength features that acts as a polarizing beam splitter", Appl. Optics, vol. 40, no. 32, pp. 5898-5905, 2001.

[3] G. H. Dammann and K. Görtler, "High efficiency in-line multiple imaging by means of multiple phase holograms", Optics Commun., vol. 3, no. 5, pp. 312-315, 1971.

[4] H. Dammann and E. Klotz, "Coherent optical generation and inspection of two-dimensional periodic structures", Opt. Acta, vol. 24, no. 4, pp. 505-515, 1977.

[5] F. Gori et al., "Analytical derivation of the optimum triplicator", Optics Commun., vol. 157, no. 1-6, pp. 13-16, 2008.

[6] R. Borghi, F. Frezza, L. Pajewski, M. Santarsiero, and G. Schettini, "Optimum even-phase four-beam multiplier", Optical Engin., vol. 41, no. 11, pp. 2736-2742, 2002.

[7] J. Song, Y. Li, X. Zhou, and X. Li, "Planar grating multiplexers using silicon nanowire technology: numerical simulations and fabrications", Progress in Electromag. Res., vol. 123, pp. 509-526, 2012.

[8] J. L. Jiang, H. Wu, L. Y. Jiang, and X. Y. Li, "Genetic optimization of double subwavelength metal slits sorrounded by surface dielectric gratings for directional beaming manipulation", Optics Commun., vol. 285, no. 8, pp. 2201-2206, 2012.

[9] G. Bloom et al., "Design and optimization of a high-efficiency array generator in the mid-IR with binary subwavelength grooves", Appl. Optics, vol. 50, no. 5, pp. 701-709, 2011.

[10] F. Wyrowski, "Upper bound of the diffractive efficiency of diffractive phase elements", Opt. Letters, vol. 16, no. 24, pp. 1915-1917, 1991.

[11] U. Krackhardt, J. N. Mait, and N. Streibl, "Upper bound on the diffraction efficiency of phase-only fan out elements", Appl. Optics, vol. 31, no. 1, pp. 27-37, 1992.

[12] F. Wyrowski, "Design theory of diffractive elements in the paraxial domain", J. Opt. Soc. of America A, vol. 10, no. 7, pp. 1553-1561, 1993.

[13] J. Tervo and J. Turunen, "Paraxial-domain diffractive elements with $100 \%$ efficiency based on polarization gratings", Opt. Lett., vol. 25 , pp. 785-786, 2000.

[14] R. Borghi, F. Frezza, L. Pajewski, M. Santarsiero, and G. Schettini, "Full-wave analysis of the optimum triplicator", J. Electromag. Waves Appl., vol. 15, no. 6, pp. 689-708, 2001.

[15] F. Aroca and I. Moreno, "Comparison and experimental realization of different phase only grating designs and optimal triplicators", Optica Pura y Aplicada, vol. 49, no. 3, pp. 155-166, 2016.

[16] M. Born and E. Wolf, Principles of Optics, 7th ed. Cambridge University Press, 1999.

[17] F. Frezza, L. Pajewski, and G. Schettini, "Characterization and design of two-dimensional electromagnetic band-gap structures by use of a full-wave method for diffraction gratings", IEEE Trans. Microwave Theory Techniq., vol. 51, no. 3, pp. 941-951, 2003.

[18] G. Zheng et al., "Analysis of finite periodic dielectric gratings by the finite-difference frequency-domain method with the sub-entiredomain basis functions and wavelets", Progress in Electromag. Res., vol. 99, pp. 453-463, 2009.

[19] J.-J. Liau et al., "A new look at numerical analysis of uniform fiber Bragg gratings using coupled mode theory", Progress in Electromag. Res., vol. 93, pp. 385-401, 2009.

[20] N.-H. Sun et al., "Numerical analysis of apodized fiber Bragg gratings using coupled mode theory", Progress in Electromag. Res., vol. 99, pp. 289-306, 2009.

[21] J. Frances, C. Neipp, A. Marquez Ruiz, A. Belendez, and I. Pascual, "Analysis of reflection gratings by means of a matrix method approach", Progress in Electromag. Res., vol. 118, pp. 167-183, 2011. 
[22] F. Frezza, L. Pajewski, and G. Schettini, "Fast and accurate modeling of finite-thickness 2D-EBG structures made by circular-section rods", Microwave and Optical Technol. Lett., vol. 39, no. 6, 433-437, 2003.

[23] F. Frezza, L. Pajewski, and G. Schettini, "Periodic defects in 2DPBG materials: Full-wave analysis and design", IEEE Trans. Nanotechnol., vol. 2, no. 3, pp. 126-134, 2003.

[24] F. Frezza, L. Pajewski, and G. Schettini, "Fractal two-dimensional electromagnetic band-gap structures", IEEE Trans. Microwave Theory Techniq., vol. 52, no. 1, pp. 220-227, 2004.

[25] F. Frezza, L. Pajewski, and G. Schettini, "Numerical investigation on the filtering behavior of 2D-PBGs with multiple periodic defects", IEEE Trans. Nanotechnol., vol. 4, no. 6, pp. 730-739, 2005.

[26] F. Frezza, L. Pajewski, and G. Schettini, "Full-wave Characterization of three-dimensional photonic bandgap structures", IEEE Trans. Nanotechnol., vol. 5, no. 5, pp. 545-553, 2006.

[27] F. Frezza, L. Pajewski, E. Piuzzi, C. Ponti, and G. Schettini, "Design and fabrication of a 3D-EBG superstrate for patch antennas", in Proc. 39th Eur. Microwave Conf. EuMC 2009, Rome, Italy, 2009, pp. 1496-1499.

[28] F. Frezza, L. Pajewski, E. Piuzzi, C. Ponti, and G. Schettini, "Analysis and experimental characterization of an alumina woodpilecovered planar antenna", in Proc. 40th Eur. Microwave Conf. EuMC 2010, Paris, France, 2010, pp. 200-203.

[29] F. Frezza, L. Pajewski, E. Piuzzi, C. Ponti, and G. Schettini, "Advances in EBG-resonator antenna research", in Proc. Int. Symp. on Antennas and Propagation ISAP 2012, Nagoya, Japan, 2012, pp. 1301-1304.
[30] S. Ceccuzzi, L. Pajewski, C. Ponti, and G. Schettini, "Directive propagation in two EBG structures: a comparison", 2013 IEEE MTTS Int. Microwave Symp. Digest IMS 2013, Seattle, WA, USA, 2013, (doi: 0.1109/MWSYM.2013.6697579).

[31] S. Ceccuzzi, L. Pajewski, C. Ponti, and G. Schettini, "Comparison between two methods for directivity enhancement of antennas through 2-D EBGs", in Proc. 2013 Progress in Electromag. Res. Symp. PIERS 2013, Stockholm, Sweden, 2013, pp. 557-561.

[32] F. Frezza, L. Pajewski, E. Piuzzi, C Ponti, and G. Schettini, "Radiation-enhancement properties of an X-band woodpile EBG and its application to a planar antenna", Int. J. on Antennas and Propag., vol. 2014, Article ID 729187, 2014 (doi: 10.1155/2014/729187).

[33] S. Ceccuzzi, L. Pajewski, C. Ponti, and G. Schettini, "Directive EBG antennas: a comparison between two different radiating mechanisms", IEEE Trans. on Antennas and Propag., vol. 62, no. 10, pp. 5420-5424, 2014 (doi: 10.1109/TAP.2014.2346174).

Fabrizio Frezza - for biography, see this issue, p. 23.

Lara Pajewski - for biography, see this issue, p. 29.

Marian Marciniak - for biography, see this issue, p. 35. 